\title{
On discriminating visual textures and images
}

\author{
TERRY CAELLI \\ University of Newcastle, Newcastle, N.S.W. 2308, Australia
}

\begin{abstract}
Recent developments in modeling image discrimination by feature analytic and frequency selective methods are discussed. Some issues relating to the design of two-dimensional spatial frequency filters are developed within the context of two experiments on texture discrimination using artificial and naturally occurring textures. Results of these experiments indicate that, given an adequately formulated relationship between spatial frequency and orientation tuning parameters of the filter, one can predict a variety of texture discriminations using only amplitudespecific models. Finally, guidelines are established for ascertaining when phase transmission characteristics do become critical in two-dimensional image processing by the human observer.
\end{abstract}

Visual textures, having great variability, are discriminable for many reasons, and so any attempt to model and predict aspects of texture perception requires a sufficiently robust language to include the variability that is characteristic of what we see. The history of models and studies in visual image discrimination is replete with "context-specific" issues from questions concerning the appropriate discriminating metrics for random polygons (see Brown \& Owen, 1967) to generative grammar approaches to feature list studies of alphabetic character discrimination (see Leeuwenberg, 1978).

Yet a problem still remains: Is there such a language-a language from which we can predict human image or texture discrimination over a wide range of image classes and which is consistent with the known physiological processes of the visual system?

The aim of this paper is to group the three current approaches to this problem into a unified perspective and to show how it can be simulated in the context of digital image processing to predict discrimination between a variety of naturally occurring and artificially generated textures which may have the variability suggested above.

The first of the approaches involves the manipulation of specific features between two textured surfaces and to observe discrimination. Such feature analytic studies propose that the visual system discriminates between textures when the count of feature states between texture pairs reaches a critical difference. For example, when the orientation distribution differences are large enough, discrimination occurs. Beck (1966), Caelli and Julesz (1979), and others have shown that this model works adequately with some texture classes, particularly with textures defined on grid micropattern structures (Figure 1a) and generated from a specified feature list.

This project was supported by a grant from the Australian Research Grants Committee. I would like to thank Kim Harrison for helping in conducting these experiments.
This feature-extraction model has a space-invariance property whereby discrimination between textures is determined by statistical properties of the feature states and not by their specific absolute position within the textures. However, this property presents one major complication. Features themselves have spatial definitions that are fixed relative to each other. Line segments, angles, and edges are defined by a specific spatial cluster, or arrangement, which cannot be otherwise located. Consequently, the space-invariance property cannot precede feature definition or analysis. One clear example of the fixed nature of features is the finding of Caelli, Julesz, and Gilbert (1978) that textures with identical length distributions between disk elements (dipole length distributions) can still have perceivably different local geometric structures, which in some circumstances yield texture discrimination (Figure 1b).

Caelli and Julesz (1978) found an independent set of features (of orientation, corners and "closure") which yield texture discrimination (Figure 1). More recently, Julesz (1981) has generated a general class of feature extractors in texture discrimination (termed "textons") having these features as examples. However, the problem with generating predictions about texture discrimination from feature-extraction procedures is that of creating feature list languages that are capable of generating criteria for exhaustive enumeration. The specific aim of the Caelli and Julesz $(1978,1979)$ and Julesz (1981) work is to do this precisely. One extra problem with feature extraction is that, particularly in the context of natural scenes, it usually presumes edge extraction, since feature identification requires the observer to partition the image or texture into boundary regions which so delimit various features.

In contrast, orthogonal expansions (the breaking up of a function into a sum of independent components; see Caelli, 1981), since they entail a sum of independent terms, make it possible to draw conclusions unequivocally about the number of units involved (filter 
a

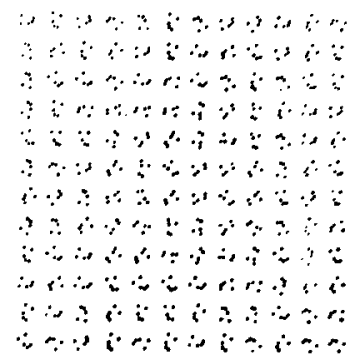

b

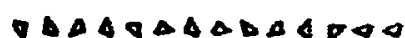

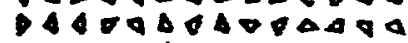
-0004070004000 - 040008000000 D OAADAF TKADAO

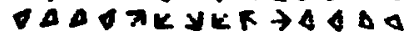

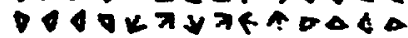

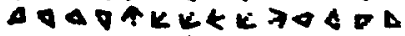

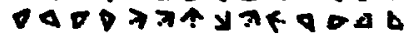

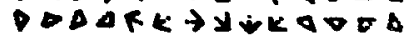
aDAOAODDAaADac -avadoboocabo

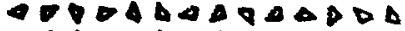
$400-040004004$

Figure 1. (a) Micropattern-based textures where texture differences are predictable from statistical propertles of the micropatterns. (b) Three types of features which yield texture discrimination even when amplitude spectra are identical (from Caelli, Julesz, \& Gilbert, 1978).

mechanisms) and image details that are adequate for picture description in a most general sense.

For these reasons, and others, recent attempts to analyze texture discrimination involve, in a very real way, the use of orthogonal decompositions of images: specifically, the Fourier series. This (second) approach states that the visual system breaks down the two-dimensional image into a set of independent components such that information in a texture at position $\left\{\mathbf{x}_{1}, \mathbf{x}_{2}\right\}$ is specified by (discrete model)

$$
\begin{aligned}
T(\underset{\sim}{\mathrm{x}})= & \sum_{\mathrm{u}_{1}, \mathrm{u}_{2}} \mathrm{~F}_{\mathrm{R}}\left(\mathrm{u}_{\mathrm{i}}, \mathrm{u}_{2}\right) \cos \left(\frac{\mathrm{u}_{1} \mathrm{x}_{1}+\mathrm{u}_{2} \mathrm{x}_{2}}{\mathrm{~N}}\right) \\
& +\mathrm{i} \sum_{\mathrm{u}_{1}, \mathrm{u}_{2}} \mathrm{~F}_{\mathrm{I}}\left(\mathrm{u}_{1}, \mathrm{u}_{2}\right) \sin \left(\frac{\mathrm{u}_{1} \mathrm{x}_{1}+\mathrm{u}_{2} \mathrm{x}_{2}}{\mathrm{~N}}\right)
\end{aligned}
$$

or

$$
\mathrm{T}(\underset{\sim}{\mathrm{x}})=\sum_{\mathrm{u}_{1}, \mathrm{u}_{2}} \mathrm{~A}\left(\mathrm{u}_{1}, \mathrm{u}_{2}\right) \exp \left\{\mathrm{i}\left[\phi\left(\mathrm{u}_{1}, \mathrm{u}_{2}\right)+\varrho\left(\mathrm{x}_{1}, \mathrm{x}_{2}, \mathrm{u}_{1}, \mathrm{u}_{2}\right)\right]\right\} .
$$

Here $A\left(u_{1}, u_{2}\right)$ is the amplitude spectrum defined by

$$
A\left(u_{1}, u_{2}\right)=\left[F_{R}^{2}\left(u_{1}, u_{2}\right)+F_{I}^{2}\left(u_{1}, u_{2}\right)\right]^{1 / 2}
$$

and $\phi\left(u_{1}, u_{2}\right)$ is the phase spectrum defined by

$$
\phi\left(\mathrm{u}_{1}, \mathrm{u}_{2}\right)=\tan ^{-1}\left[\mathrm{~F}_{\mathrm{I}}\left(\mathrm{u}_{1}, \mathrm{u}_{2}\right) / \mathrm{F}_{\mathrm{R}}\left(\mathrm{u}_{1}, \mathrm{u}_{2}\right)\right] .
$$

$F_{R}$ and $F_{I}$ correspond to the real and imaginary components of Equation 2. $A^{\prime}$ and $\phi^{\prime}$ represent the modulated amplitude and phase spectra representing the transfer characteristics of the visual system. That is, the transfer functions $\mathrm{G}$ and $\mathrm{H}$ defined by

$$
\begin{aligned}
& A^{\prime}\left(\mathrm{u}_{1}, \mathrm{u}_{2}\right)=\mathrm{G}\left(\mathrm{u}_{1}, \mathrm{u}_{2}\right) \cdot A\left(\mathrm{u}_{1}, \mathrm{u}_{2}\right) \\
& \phi^{\prime}\left(\mathrm{u}_{1}, \mathrm{u}_{2}\right)=\mathrm{H}\left(\mathrm{u}_{1}, \mathrm{u}_{2}\right) \cdot \phi\left(\mathrm{u}_{1}, \mathrm{u}_{2}\right),
\end{aligned}
$$

code, in a quantitative way, the loss of amplitude or phase (position) information in the system (Caelli, 1981). Finally,

$$
\varrho\left(x_{1}, x_{2} ; u_{1}, u_{2}\right)=\frac{2 \pi}{N}\left(u_{1} x_{1}+u_{2}, x_{2}\right)
$$

represents the "transmission line" or allocation procedure for reconstructing the image on an $\mathbf{N} \times \mathbf{N}$ grid (position code) in terms of the amplitude and phase coefficients of the expansion.

Since $\left\{\mathrm{u}_{1}, \mathrm{u}_{2}\right\}$ correspond to the vertical and horizontal spatial frequency components of the image (texture), various authors argue for different forms of $G\left(u_{1}, u_{2}\right)$ in terms of the number of frequency channels and their sensitivity profiles. Figure 2 shows some examples based on the known threshold sensitivity of the human visual system to one-dimensional sinusoidal gratings.

A third approach to such texture coding problems 

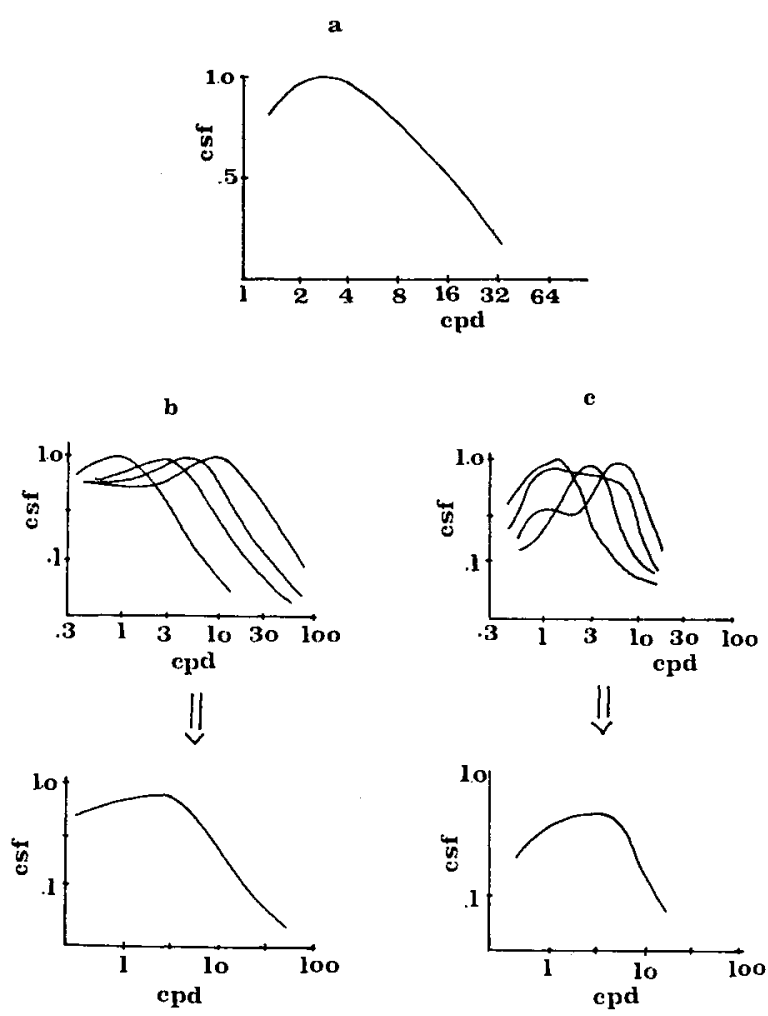

Figure 2. (a) Typical human contrast sensitivity function for onedimensional gratings. (b) Four-channel filter model of Mostafavi and Sakrison (1976). (c) Four-channel model of Richards and Polit (1974). The notation csf refers to contrast sensitivity (arbitrary units), and the abscissa indicates spatial frequency in cycles per degree (cpd). The bottom-line curves refer to average tuning characteristic of the channels.

is that originated by Julesz (1962) and developed by Caelli and Julesz (1978), Caelli, Preston, and Howell (1978), and Uttal (1975). These authors argue that the visual system extracts specific relational information from an image in order to discriminate, extract contours, etc. Of prominent importance is the dipole, or second-order statistics, corresponding to the length and orientation distributions of interelement connections. For black-and-white images, this corresponds, in turn, to the autocorrelogram of an image, and, since two images with the same autocorrelograms have identical amplitude spectra (Equation 2), there is a formal correspondence between this third approach and the Fourier model (Julesz \& Caelli, 1979). The main difference between these models is precisely the definition of the filtering mechanisms, both in terms of amplitude and phase processing in the visual system.

In summary, we are concerned with the Fourier model for the following reasons: (1) The Fourier amplitude spectrum is directly related to an image's autocorrelogram; (2) in principle, the model is exhaustive-when properly developed in its two- dimensional amplitude and phase transfer characteristics; (3) the system (Equation 1) is simply defined by the transfer functions (Equation 4). Specifically, the aim of this project was to evaluate recent attempts to apply this language to image discrimination, and so to develop in more detail the two-dimensional transfer functions (Equation 4).

\section{Recent Applications of the Fourier Model to Image Discrimination}

The works of Coffin (1978) and Harvey and Gervais (1978) provide two good examples of this model and, by the nature of their contradictory results, illustrate the limitations of the language.

Case 1: The Harvey and Gervais study. By ignoring phase, orientation, and the implicit two-dimensional nature of visual textures, these authors argued that texture discrimination could be predicted by the difference in activity generated in a number of spatial frequency channels by two textures. They specifically used the four-channel case, having transfer functions defined by (1) Richards and Polit (1974), (2) Mostafavi and Sakrison (1976)-exponential, or (3) Butterworth filters (Gaussians), also developed by Mostafavi and Sakrison (1976): Figure 2. These authors cleverly formulate the problem in two different ways via discriminant analysis and multidimensional scaling.

The discriminant analysis model argues that, with four channels, the linear equations of the form:

$$
D=\sum_{i=1}^{4} \omega_{i} D_{i}
$$

maximize the differences between texture groupings. Here, $D_{i}$ corresponds to the output of channel $i$ and $\omega_{i}$ corresponds to the regression coefficients of the discriminant function (Cooley \& Lohnes, 1971). The authors demonstrated (by a sorting task in which the similarity measure between two textures was determined by the number of times two textures were placed in the same group) that the Richards and Polit filter profiles (Figure 2) predicted discrimination best of all.

Similarity between texture pairs was also defined in terms of proximity in a multidimensional (Euclidean) space, and multidimensional scaling was enacted on the judged texture similarity matrix (the MDSCAL routine; see Kruskal, 1964). In addition, factor analysis and canonical correlations were used to separate out and identify the sources of variance. All in all, results indicated that the two main channels of Richards and Polit (for the specific values chosen)-high spatial frequency $\left(\mathrm{H}\right.$ : pecking at about $\left.8 \mathrm{cpd}^{1}\right)$ and visibility (V: middle frequency range pecking at about $3 \mathrm{cpd}$ ) -could explain $89.1 \%$ of the variance.

In relation to the initial formulation (Equations 2-4), this work suggests that, for "one-dimensional" 
textures, ${ }^{2}$ the visual system processes the amplitude coefficients of the Fourier series in a way consistent with the summation of outputs from a set of channels having the individual and net frequency modulation characteristics shown in Figure 2. That is, due to the linearity of amplitude, filtering the net output of the Richards and Polit filters is determined by their sum (Figure 2):

$$
\mathrm{G}_{\mathrm{T}}=\sum_{\mathrm{i}=1}^{4} \mathrm{G}_{\mathrm{i}} \text {. }
$$

However, when strictly two-dimensional textures (that is, images whose spatial information is not defined by a one-dimensional waveform) are of interest, the above formulation is inadequate. The visual filtering mechanisms must entail orientation/frequency and phase modulation transfer characteristics not contained in these more simple filters.

Case 2: The Coffin study. In contrast to the positive results of Harvey and Gervais, Coffin (1978) was not very successful in predicting confusability (or similarity) of alphabetic characters via Fourier spectra correlation measures. If we regard character discrimination in a way similar to pattern recognition or pattern detection, we may well conclude with Coffin that feature extraction and cognitive strategies are more appropriate than Fourier methods. However, it suffices to note, at this point, fundamental differences between the model testing technologies in each study.

In the Coffin study, alphabetic characters were digitized and Fourier analyzed according to the usual fast Fourier transform methods (see Caelli, 1981). Coffin then calculated a series of between-character correlations (Pearson's $r$ and Spearman's rho) in terms of amplitude spectra, modulated amplitude spectra, full Fourier transforms, and even "raw-letter" correlations. These various correlations were, in turn, correlated with past experimental findings on character confusions.

The results indicated that such spectra correlations were not strong predictors (via correlation) of character confusability - whether the spectra be filtered or not by the contrast sensitivity function shown in Figure 2a. However, three points need to be made before concluding that the model does not work for character discrimination. First, intercorrelations between various experimental results are not always high. For example, Coffin reports a highest correlation of .62 between character confusion results collected by different experimenters. Secondly, the relative positions of the characters-their overlap characteristics-affect their phase spectra relationships, and since Coffin was cross-correlating their full spectra, this must be considered, but was not. Finally, and most importantly, to simulate the type of information processing that occurs in the visual system during two-dimensional character discrimination, orientation, and its relationship to spatial frequency and phase, should be dealt with.

Due to the one-dimensional nature of the Harvey and Gervais textures and the difficulties found with alphabetical characters in the Coffin study, it is still not possible to clearly evaluate the role of Fourier transform methods of defining two-dimensional image discrimination. In the following section, general properties of a two-dimensional filter are considered as a generalization of the one-dimensional types already discussed.

\section{Exploring One Type of Two-Dimensional Filter: The "Clam-Shell' Filter}

Here the components of the amplitude/phase spectra are considered as being subject to frequency and orientation analysis.

When defining the types of amplitude and orientation information transmission in the visual system by the Fourier model, it should be noted that the system is assumed to be linear such that the information modulation characteristics of each channel or unit additively contribute to a total frequency transfer function (Figure 2). In particular, with amplitude modulation, the types of channel properties suggested by Figure 2 result in an amplitude-specific modulation transfer function (MTF) of the general form

$$
\operatorname{MTF}(\omega)=k \omega^{\alpha-1} \exp (-\omega / \beta),
$$

where $\omega$ corresponds to the (radial) spatial frequency $\omega=\sqrt{v_{1}^{2}+v_{2}^{2}}, \alpha, \beta$ being constants estimated from the human contrast sensitivity function (CSF). Typically,

$$
\operatorname{MTF}(\omega)=K_{A} \omega^{1.8} \exp (-0.6 \omega),
$$

which peaks at about $3 \mathrm{cpd}$. As a first attempt, we assume radial symmetry (frequency is modulated equally in all orientations processed) in Equation 8. Orientation selectivity is defined by: (a) specifying the number of orientation detectors (channels) at each frequency range being delimited (in its upper bound) by the vernier and line acuity of the visual system, and (b) determining the response or tuning width of each orientation detector as a function of (a) and spatial frequency.

To model these characteristics, the following filter was developed. Consider an arbitrary position in the two-dimensional spatial frequency spectrum: $\left(\mathrm{u}_{1}, \mathrm{u}_{2}\right)$. It has an orientation $\theta^{*}=\tan ^{-1}\left(u_{2} / u_{1}\right)$ and must lie on, or between, two orientation detector peak response angles (Figure 3): $\theta_{\mathrm{L}}$ and $\theta_{\mathrm{u}}$. Consequently, the perpendicular projection distances of the point of angle $\theta^{*}$ to each of these orientations are:

$$
\begin{aligned}
& d_{u}=\omega \sin \left(\theta_{u}-\theta^{*}\right) \\
& d_{L}=\omega \sin \left(\theta^{*}-\theta_{L}\right) .
\end{aligned}
$$


These lengths are then weighted by Gaussian orientation tuning curves of the form

$$
\frac{1}{\sigma(\omega) \sqrt{2 \pi}} \exp \left\{-\left[d_{i} / \sigma(\omega)\right]^{2}\right\}
$$

where variances modulate as a linear function of spatial frequency

$$
\sigma(\omega)=\gamma \omega+\delta
$$

Here specific information is required concerning the relationship(s) between spatial frequency and orientation tuning characteristics before the full "clamshell" filter can be defined. Clearly, the feasible candidates are: As spatial frequency increases (a) orientation tuning decreases, or (b) it increases (Figure 3).

As is implicit in Figure 3, both the tuning widths and number of these orientation detectors could change as a function of spatial frequency. These possible relationships will be experimentally investigated, to a limited extent, in Experiment 1, but have been directly investigated in two other experiments by the author (Caelli, Note 1; Caelli \& Harrison, Note 2). For the moment, it should be noted that, since Equation 10 operates only between neighboring orientation detectors, the orientation response (orientation sensitivity component) is derived from the sum:

$$
\operatorname{MTF}_{\theta}\left(\theta^{*}, \omega\right)=\mathrm{k}_{\mathrm{o}} \sum_{i=10}^{2} \frac{1}{\sigma(\omega)} \exp \left\{-\left[\mathrm{d}_{\mathrm{i}} / \sigma(\omega)\right]^{2}\right\}
$$

This is not to imply that orientation interactions, in this case summation, occur only between neighboring orientation detectors. Rather, they were chosen, in this case, simply because the Gaussian tuning characteristics implied that almost all the orientation coding would be done this way. In fact, Caelli and Harrison (Note 2) have developed a digital version of this analog filter whereby this form of orientation coding operation can be avoided while still retaining the use of a finite number of orientation detectors having tuning widths that vary as a function of spatial frequency.

Combining both orientation and amplitude response functions results in the amplitude-specific transfer function

$$
\operatorname{MTF}(\theta, \omega)=\operatorname{MTF}_{\mathbf{A}} \cdot \operatorname{MTF}_{\theta}
$$

defined by Equations 9 and 13 above. This constitutes the "clam-shell" shape illustrated in Figure 3.

The second component-that of phase-in Equation 4 is more difficult to define. Whereas the image amplitude spectrum codes the relative strengths of the frequency and orientation components present,
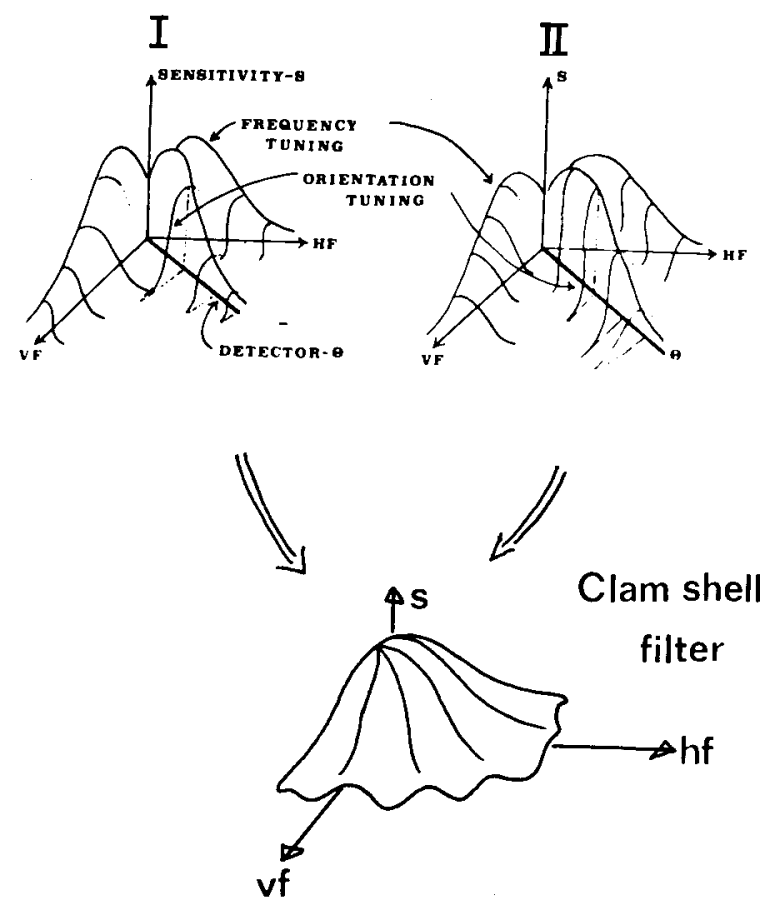

Figure 3. The "clam-shell" filter representing the modulation transfer characteristics for the processing of amplitude information as a function of spatial frequency and orfentation channels. Case 1 lllustrates a decrease in orientation tuning as frequency increases, and Case 2, the opposite situation. Note that these cases assume a constant number of orlentation detectors, and, in general, more overlap occurs in the lower frequency range.

the phase spectrum codes the relative position of such components in the image. In this sense, the phase spectrum "contains the image," since it codes the juxtapositions of objects, edges, etc. For this reason, Caelli and Bevan (Note 3 ) recently proposed that phase filtering is more properly defined by the phase resolution, or position sensitivity of the visual system to different frequency/orientation components in the image. In particular, Caelli and Julesz (1978) have shown that specific types of phase differences between two textures can yield texture discrimination even when their amplitude spectra are identical (see Julesz, 1981, for a review).

In a recent paper, Brettel, Caelli, Hilz, and Rentschler (1981) further explored the issue of two-dimensional phase modulation in the visual system. However, for the present analyses, we are concerned exclusively with the filter profiles for amplitude processing in the visual system, and so I have endeavored when possible sible to standardize phase properties of the images.

\section{EXPERIMENT 1 \\ FREQUENCY (SIZE)/ORIENTATION TUNING AND TEXTURE DISCRIMINATION}

As is clear from the filter profiles discussed in Figure 3, it is necessary to determine orientation sensitivity (tuning) as a function of spatial frequency 
or size before the two-dimensional filter profile can be established. Although there is evidence from electrophysiological studies (see De Valois, 1978) that orientation tuning varies with spatial frequency, the only clear psychophysical evidence, to this stage, comes from orientation illusion (adaptation) effects (Movshon \& Carpenter, 1973; Caelli, Note 1). Here, the results indicate that as grating spatial frequency increases, the orientation illusion angular range decreases. Presuming a direct relationship between adaptation effects and normal orientation sensitivity, these results indicate that as spatial frequency increases, either (a) the orientation tuning width decreases and/or (b) the orientation detector separation increases-implying the presence of fewer detectors, so inhibiting interaction effects reflected by increased illusory effects.

However, these results come from spatially overlapping sources and not the spatially distributed orientations that occur in natural images or textures. So, in this experiment, a form of orientation/frequency tuning will be examined within the context of texture discrimination, using artificially generated textures.

As illustrated in Figure 4, a texture pair consisted of two identical (in range) uniform distributions of oriented rectangles. For fixed orientation ranges and sizes, the two distributions are then pulled apart until the observer detects a difference between the textures. In this way, the orientation sensitivity can be established for various size elements (and so, spatial frequency bands) and orientation bands. This form of orientation/frequency sensitivity can then be applied directly to natural texture discrimination (in Experiment 2).

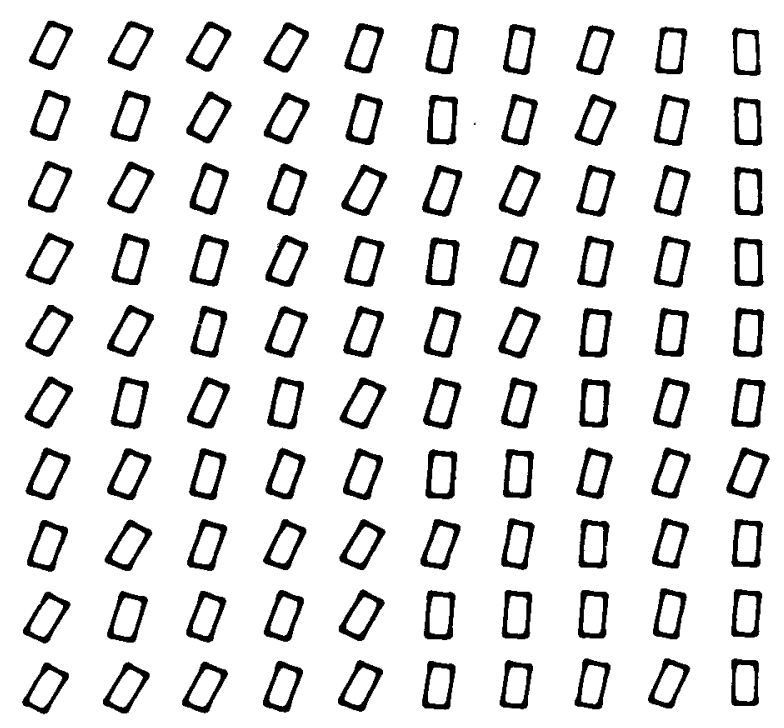

Figure 4. Textured stimuli used In Experiment 1. Here both textures have identically sized rectangles and identical orientation ranges, yet shifted 10 deg apart (left: 60-80 deg; right: 70-90 deg).

\section{Method}

Subjects. The observers consisted of four undergraduate students at the University of Newcastle who were naive to the purposes of the experiment. All were emmetropic and, due to the large number of sessions and trials, were paid for their services.

Stimulus and Apparatus. Texture pairs were generated by a PDP-11/40 computer and vector-generator system and were displayed on an HP1310A CRT at an exposure time of $100 \mathrm{msec}$. Rectangular micropatterns were chosen for the following reason. A size factor was required to investigate frequency/orientation effects, but, due to the large number of vectors needed for display in such a brief period ( 400 vectors in Figure 4), only outlines could be used. Fortunately, the relationship between the rectangular dimensions $(\alpha$, longer vertical component; $\beta$, smaller horizontal: Figure 4) and texture amplitude spectra can be analytically determined by

$\mathrm{F}\left(\mathrm{u}_{1}, \mathrm{u}_{2}\right)=\sum_{\mathrm{i}=1}\left[\frac{\mathrm{k}\left(1-\mathrm{k}^{2}\right)^{1 / 2} \sin \left(\beta \mathrm{u}_{2}-\alpha \mathrm{u}_{1}\right)-\mathrm{k}^{2} \cos \left(\beta \mathrm{u}_{2}-\alpha \mathrm{u}_{1}\right)}{\mathrm{u}_{1} \mathrm{u}_{2}}\right] \theta_{\mathrm{i}}$

where $\left(u_{1}, u_{2}\right)$ corresponds to the horizontal and vertical frequency components, $k=\sin \left(\delta \alpha \cdot u_{1}\right)=\sin \left(\delta \beta \cdot u_{2}\right)$ corresponds to the rectangular boundary width, and $-\theta_{\mathrm{i}}$ corresponds to the orientation of the rectangular segment. This result comes from three properties of the Fourier transform (see Caelli, 1981) amplitude spectrum: (a) shift invariance, (b) a rotation $\theta$ in the image domain that corresponds to a rotation of $-\theta$ in the spectrum, and (c) linearity, the sum of the Fourier transforms of each micropattern equals the Fourier transform of the full texture. As can be seen from Equation 15, increasing the rectangular dimensions increases the upper frequency components (relative to the lower).

Finally, the average luminance of each display was $4 \mathrm{~cd} / \mathrm{m}^{2}$, and the texture parameters being varied were: (a) size and aspect ratios of rectangular micropatterns, each texture consisting of $10 \times 5$ rectangles (100 in total per display), there being five cases, measuring $12 \times 2 \mathrm{~min}, 8 \times 2 \mathrm{~min}, 6 \times 2 \mathrm{~min}, 12 \times 4 \mathrm{~min}, 8 \times 4 \mathrm{~min}$, and $7 \times 4 \mathrm{~min}$ of visual angle (Figure 4); and (b) orientation range, with rectangular texture pairs being generated such that the orientation distributions of each texture were equivalent uniform distributions within 2-, 5-, 10-, 20-, 40-, and 80-deg ranges. Initially, both were centered at about $90 \mathrm{deg}$ (the catch trial condition). Under program control, one texture (chosen randomly) could be altered to have the same uniform distribution about another angle-that is, the texture orientation distributions could be separated incrementally in order to determine the critical shift value for discrimination.

Procedure. Each observer was run over six different sessions, corresponding to the six different rectangular sizes. For a given orientation range, a random-staircase procedure was used, whereby the various separation angles between the distributions were arranged over trials. On each trial, the subjects were asked to fixate on a center disk and were then presented the texture pair for $100 \mathrm{msec}$, after which they were asked to respond as to whether they were the same or different (and to guess if not sure). Each subject received four presentations of each staircase for every experimental condition.

\section{Results}

In order to compare across rectangular dimensions (sizes) and the orientation ranges used, the following conversion was employed. The shift angle (that is, the angular difference between the two distributions: center-to-center) required for discrimination was divided by the particular orientation range to determine an orientation threshold in terms of the range: threshold-range unit (Figure 5). These values were calculated for all orientation ranges and rectangular sizes over replications and observers. For example, a 


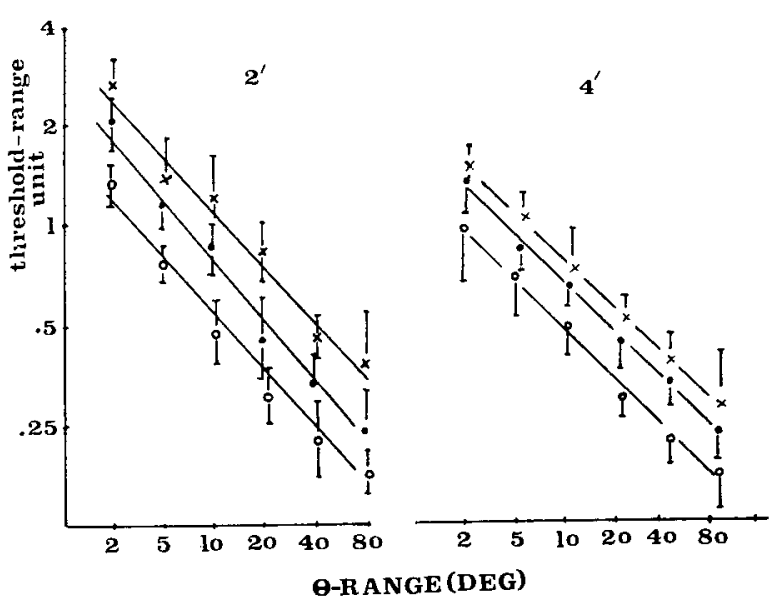

Figure 5. Average (including standard deviations) shift values (ordinate: threshold-range units), in terms of orlentation range units, required for discrimination as a function of orientation range (abscissa) and rectangular dimensions. Widths: $2 \mathrm{~min}, 4 \mathrm{~min}$; heights: $0-12 \mathrm{~min},-8 \mathrm{~min}, \times-8 \mathrm{~min}$. Regression lines shown.

range unit threshold of .5 with the $10 \mathrm{deg}$ range indicated that the two distributions had to be separated by, on average, $5 \mathrm{deg}$ before discrimination could occur. A simple three-way repeated measures analysis of variance was computed on these data [height $x$ width $\times \theta$ (range) $\times$ subjects], resulting in significant height $[\mathrm{F}(2,6)=12.11, \mathrm{p}<.05]$ and $\theta$ (range) $[\mathrm{F}(5,15)=$ $18.41, p<.05]$ effects. All other effects were insignificant $(\mathrm{p}<.05)$.

These data indicate that, as the rectangular length dimension increases, the orientation difference threshold decreases, while the width parameter (being less than the length) did not significantly affect discrimination performance. The relationship between these rectangular parameters and the amplitude/spatialfrequency spectrum is defined by Equation 15. For all rectangularly shaped images, there is a constant reciprocal relationship between amplitude and spatial frequencies of $1 / u_{1} u_{2}$. However, on top of this, the actual border thickness parameter $(\mathbf{k})$ and the perimeter length parameters $(\alpha, \beta)$ also contribute to the spectrum. As $\alpha>\beta$, the numerator of Equation 15 introduces less amplitude attenuation at the highfrequency end than it does at the low-frequency end, as a function of the total perimeter $[2(\alpha+\beta)]$. In other words, the main determinant of the high-frequency components is the total amount of illuminated border, while the "rectangularity" is largely contained in the low-frequency and phase spectra (high-frequency components increase with the number or amount of thin lines). Although not statistically significant, the increase in rectangular width did consistently decrease the orientation thresholds, and, possibly, the lack of significance in comparison with the length parameter was simply due to its relatively smaller contribution to the total perimeter.
Consequently, these results suggest that as the highfrequency components increase, orientation sensitivity increases. Such increases in sensitivity (or texture discrimination) could be related to either: Case 1increasing separation between detectors (fixed tuning width) as frequency increases-or Case 2-same separation (or less) and narrowing of the tuning response width (Figure 6).

This former option seems unlikely to be due to the fact that orientation discrimination is dependent on image clarity (or edge effects), so more orientation detectors (per unit visual angle), rather than fewer, would be expected at the upper frequency end of spatial-frequency sensitivity. Consequently, the more likely explanation of these data is illustrated by the filter described in Figure 6: Case 2. Here orientation tuning is seen to decrease as frequency increases, so making orientation acuity higher at the high-frequency end, while also decreasing the overlap characteristics which would result in higher discrimination thresholds.

These types of tuning characteristics would explain the rather strange (but consistent) result that shifts of up to 4 orientation range units were required before discrimination occurred with the very small rect-
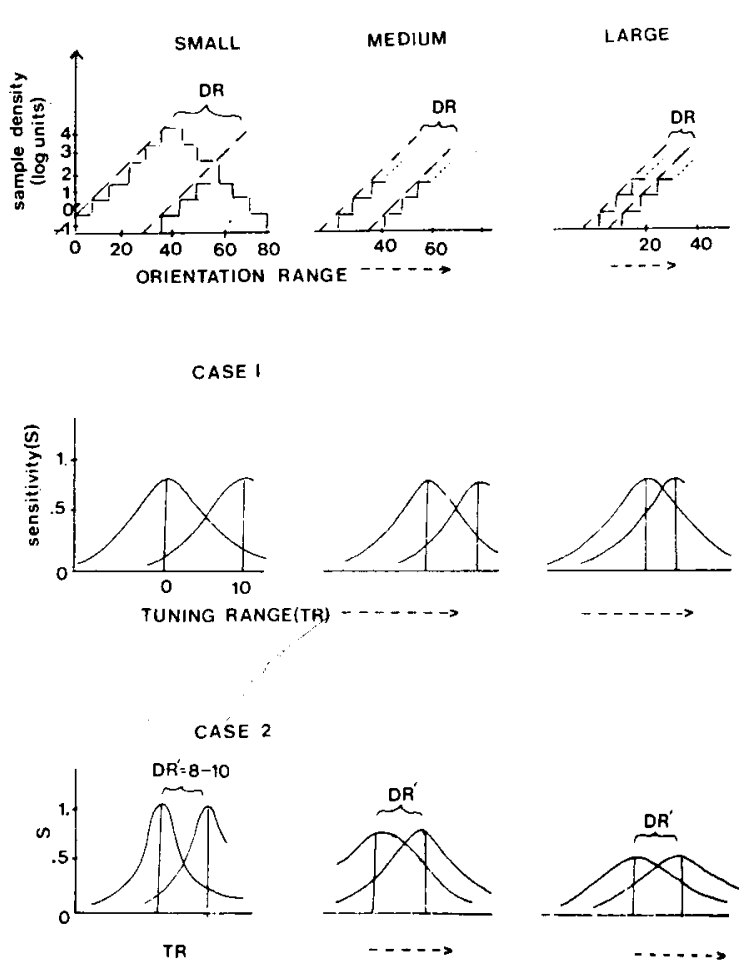

Figure 6. Top line of distributions illustrate the actual rectangular distribution shift differences (DR) which ylelded discrimination for each size of rectangle. Case 1: discrimination predicted from orientation detectors, which increase in number as frequency decrease-tuning constant. Case 2: discrimination predicted from orlentation detectors whose tuning narrows as frequency increases (higher sensitivity as frequency increases). 
angles of narrow orientation ranges, while only .125 shifts were required to yield equivalent discrimination with broad orientation ranges and larger objects. In terms of the two-dimensional filter described in Equations 10 and 13; the Case 2 profiles suggested in Figure 6 would yield as a first approximation of $\sigma(\omega)$

$$
\sigma(\omega)=5-0.125 \omega
$$

showing a narrowing of orientation tuning with increases in spatial frequency up to $32 \mathrm{cpd}$, where $\sigma(32)=1 \mathrm{deg}$.

Consistent with the aims of this project, in the following experiment such filter structures are applied to the prediction of discrimination between more naturally occurring scenes.

\section{EXPERIMENT 2 \\ DISCRIMINATING NATURAL TEXTURES AND THE FILTER PREDICTIONS}

From Experiment 1, clear evidence has been found for orientation tuning's being determined by the larger rectangular axis length, indicating that orientation sensitivity is subject to spatial summation and increases as the pertinent frequencies increase. The aim of the following experiment was to explore the applications of the filter mechanism discussed, in conjunction with the results of the foregoing experiment, for predicting the discriminability of naturally occurring textures.

\begin{abstract}
Method
Subjects. Twenty subjects were used in this experiment. All had normal or corrected-to-normal vision, and all were undergraduates of the Department of Psychology at the University of Newcastle.

Stimuli and Apparatus. The stimuli set consisted of two groups of five textures, as shown in Figure 7. These were set on flat cardboard for display in a Gerbrands three-channel tachistoscope. Each texture subtended a visual angle of $4 \mathrm{deg}$ and had a space average luminance of $4 \mathrm{~cd} / \mathrm{m}^{2}$. Reaction time equipment was included.

Procedure. Each subject was informed that the experiment was concerned with the limits of (natural) texture discrimination. They were shown one texture for $100 \mathrm{msec}$ and then, $2 \mathrm{sec}$ later, another texture for $100 \mathrm{msec}$. This 2 -sec interstimulus interval was employed to ensure that persistence of the first display had decayed past half-amplitude by the time the second texture was presented. The subjects were instructed to respond as quickly as possible as to whether the two textures were the same or different. They were told that some texture pairs would be identical and others would not; however, so that they could set their own response criteria, they were not told what percentage of catch trials was used $(17 \%)$. The 10 texture pairs and 2 catch trials were repeated three times per subject-a total of 36 presentations per texture group. Different and same (textures 2, 4 in each set) pairs were presented in random order over the 36 trials.
\end{abstract}

\section{Results}

Discrimination matrices are shown in Table 1 for both sets of stimuli. Although these data indicate some asymptotic behavior, they still yield some inter- item variance amenable to analysis. However, before proceeding further, it is necessary to describe the digital analyzing system employed.

Images are digitized, via a video camera, into a $512 \times 512$ matrix with $16^{\circ}$ (equally spaced) intensity levels. The 4-bit pixel digitized image is stored as a
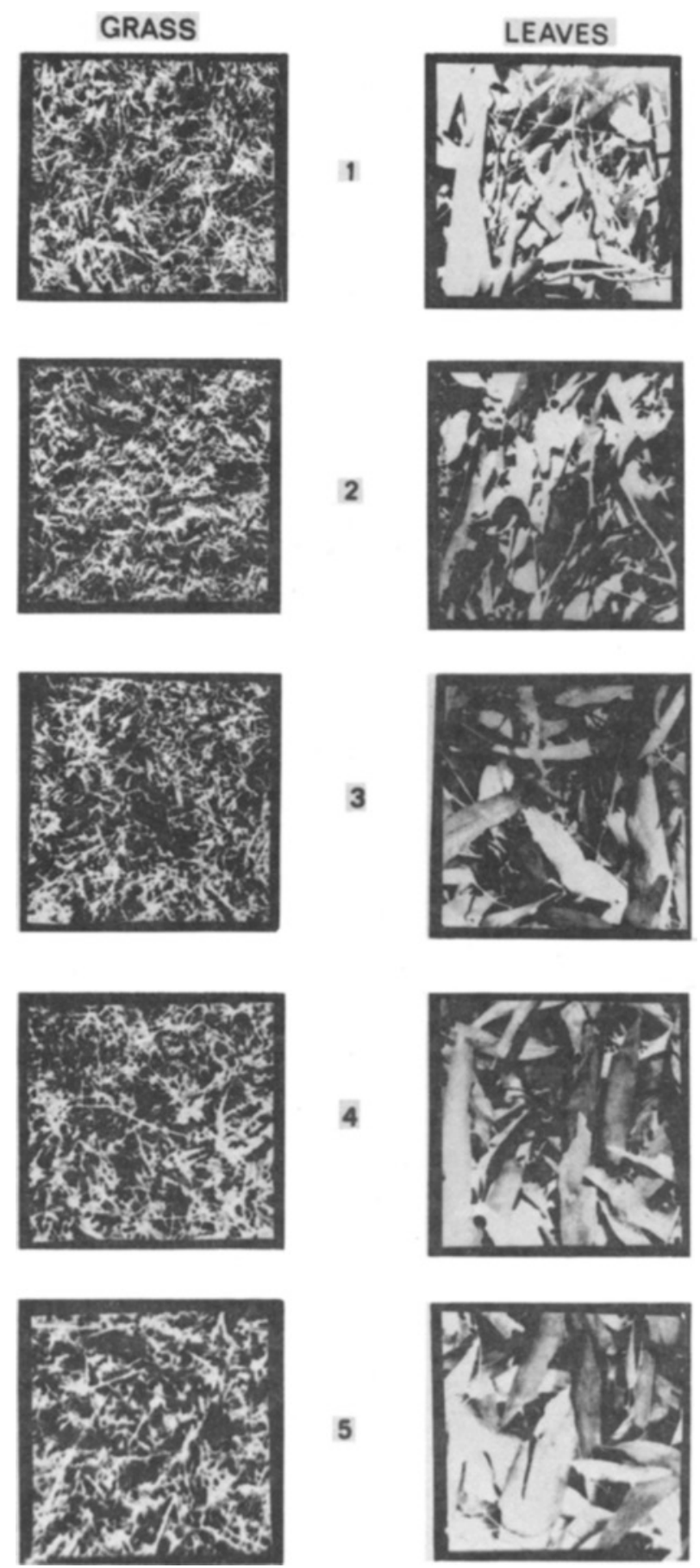

Figure 7. Natural textures used in Experiment 2 and digitized to contain up to 32 cpd of spatial frequency information. 
Table 1

Discrimination Responses (Upper Triangular Matrices) on Leaf and Grass Textures Over Subjects and Mean Reaction Times (in Milliseconds; Lower Triangular Matrices)

\begin{tabular}{|c|c|c|c|c|c|c|c|c|c|c|c|}
\hline \multicolumn{6}{|c|}{ Leaves } & \multicolumn{6}{|c|}{ Grass } \\
\hline & 1 & 2 & 3 & 4 & 5 & & 1 & 2 & 3 & 4 & 5 \\
\hline & & 80 & 83 & 90 & 100 & 1 & & 43 & 56 & 83 & 93 \\
\hline$?$ & 890 & 33 & 87 & 97 & 97 & 2 & 990 & 24 & 66 & 100 & 93 \\
\hline 3 & 860 & 830 & & 33 & 40 & 3 & 950 & 930 & & 40 & 87 \\
\hline$t$ & 890 & 870 & 850 & 13 & 90 & 4 & 940 & 960 & 870 & 17 & 67 \\
\hline 5 & 800 & 760 & 790 & 840 & & 5 & 870 & 820 & 870 & 850 & \\
\hline
\end{tabular}

Note-Catch trial error rates are illustrated in the diagonals.

256-block record on an RKOS disk unit, all controlled by a PDP-11/40 system. The process takes $7 \mathrm{sec}$, which is rather fast for such high-resolution digitization.

All 10 textures were digitized accordingly, and the following correlation analyses were performed (Pearson's r) on frequencies up to $32 \mathrm{cpd}$ : (a) All images were Fourier transformed, and bandpass ranges corresponding to $\langle 4,5-8,9-16,17-32 \mathrm{cpd}$ were cross-correlated for both amplitude and phase spectra. (b) Correlations were converted to $1-\mathbf{R}^{2}$, corresponding to the amount of variance not accounted for by the correlation between the images, that is, the amount of potentially discriminable information. Average $1-R^{2}$ values are shown in Figure 8 for both grass and leaf-type textures.

Two facets of these results should be noted. First, the textures all had virtually zero phase correlations $\left(1-R^{2} \cong 1.00\right)$, indicating that their position information is largely uncorrelated. Secondly, the 5-32cpd frequencies contained more potentially discriminating data than did lower range values with peak variation within the 5-8-cpd range, the grass being more variable than the leaves.

Finally, two further analyses were computed. Cross-correlations between (raw) amplitude spectra were plotted (as $1-R^{2}$ ) against percentage discrimination scores for the texture pairs (Figure 9a). Here two types of results emerge. For the leaf textures, the data are well spread and indicate a good degree of correlation between spectra correlations and discrimination. However, for the grass textures, two types of clusters emerge: those which are highly discriminable, having between $50 \%$ and $100 \%$ variances not accounted for by their correlations, and those which were found to be similar in their amplitude spectra and also not highly discriminable (Figure 9a).

These grass texture results are indicative of a logarithmic, psychometric function rather than the simple regression line (corresponding to the lower region of the function). What is more important, however, is the comparison of Figures $9 \mathrm{a}$ and $9 \mathrm{~b}$.

Figure $9 \mathrm{~b}$ illustrates the relationship between discrimination performance (PD) and the texture cor-

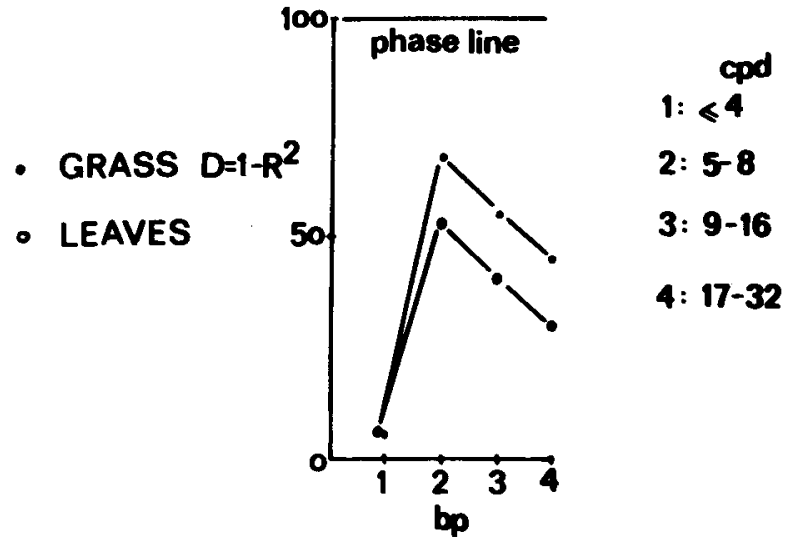

Figure 8. Amount (average percentage) of variance between the texture pairs not explained by their bandpass (bp) amplitude spectra correlations $\left[\left(1-R^{2}\right) \times 100 \times D\right]$. Phase line represents the almost zero correlation between the phase spectra for both stimulus types.
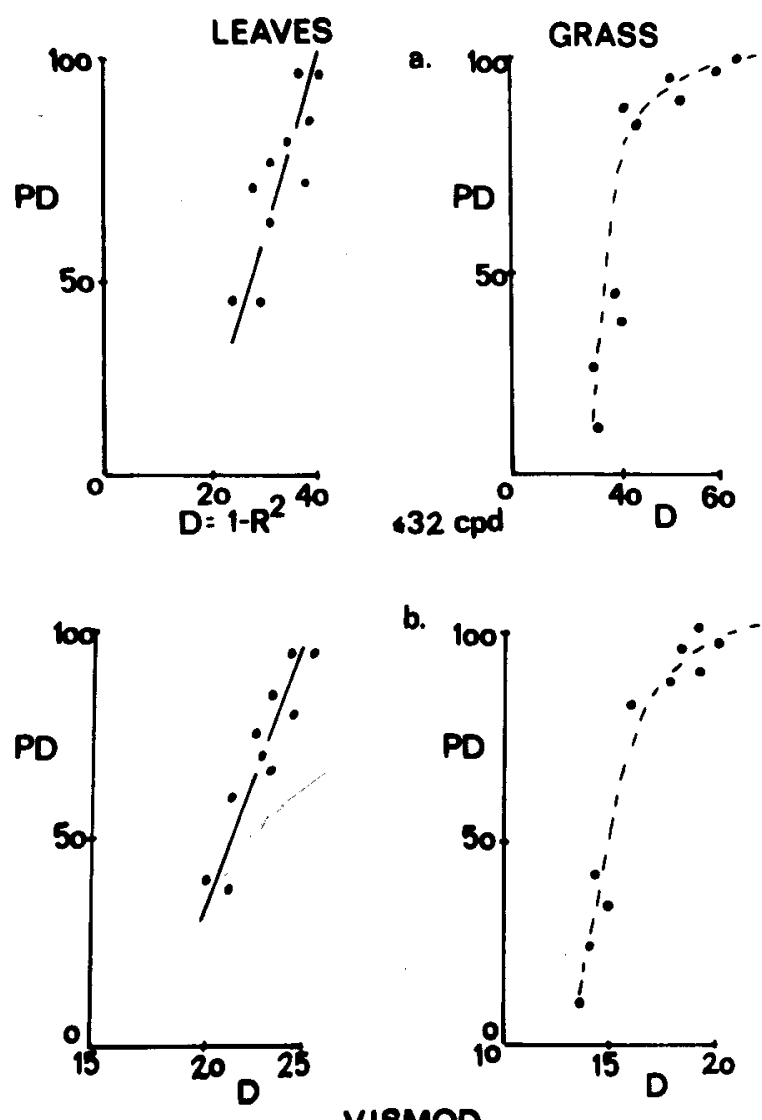

b.

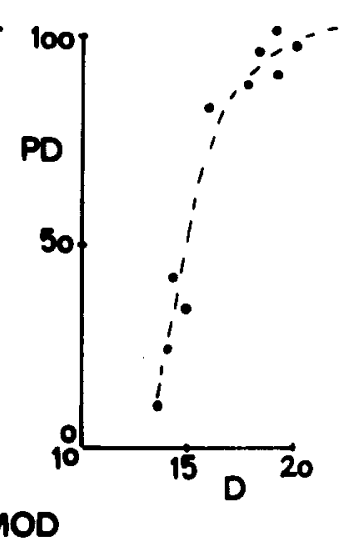

Figure 9. (a) Plot of texture discrimination performance (PD: percentage discrimination over subjects) against raw amplitude spectra correlation residuals $\left[\left(1-R^{2}\right) \times 100=D\right]$, up to $32 \mathrm{cpd}$, for both leaves and grass textures. (b) Discrimination performance plotted against texture amplitude spectra correlations realduals (D), filtered by the two-dimensional orientation/frequency clamshell filter: 36 orientation detectors. 
relations between amplitude spectra filtered by the "clam-shell" filter, which was composed of 36 orientation detectors, with the orientation/spatial frequency tuning function defined by Equations 10 and 16, frequency response defined by Equation 8 for a peak frequency at $3 \mathrm{cpd}$, and the total MTF defined by Equation 13. Although such filtering must reduce the $1-R^{2}$ measure (due to the fact that we are adding correlated information), the relationship between amplitude spectra and discrimination remains similar to the raw spectra results, the point being that the filter has ignored a significant amount of spectrum information and still has produced similar correlations.

\section{DISCUSSION}

The primary concept behind the Fourier model is that the human observer, in processing visual information, has mechanisms which decompose an image into its (sinusoidal) frequency components, which include both amplitude and phase coding. The secondary concept, or psychophysical conjecture, is that all of this objective information is (a) not coded per se, and (b) that which is coded is done so according to specific filter profiles. Consequently, the correlations observed with complete amplitude spectra, as opposed to the two-dimensional filter for discrimination performance, support the secondary conjecture. That is, the filter model (always) argues for what information is redundant in the system just as much as it argues for what is salient to the receiver.

For example, in this experiment each natural texture was digitized with $1.05 \times 10^{6}$ bits of information. The MTF with a peak frequency of $3 \mathrm{cpd}$ and 36 orientation detectors reduces this information to no more than half the amount, yet performance can be predicted just as well. Needless to say, this information can most probably be reduced further without any noticeable difference between raw amplitude spectra predictions. At the moment, however, this experiment was designed to illustrate the processes involved in the two-dimensional filter, rather than to exhaust its predictive limits (which must exist). For example, the relationship(s) between orientation tuning characteristics and the number of orientation detectors need to be explored both experimentally and theoretically at each frequency band, and, clearly, if too few detectors are employed, predictions of discrimination must decrease (see Caelli \& Harrison, Note 2).

A most important attribute of the Experiment 2 textures was that they all had approximately zero phase spectra correlations. This control induces the subject to refrain, within limits, from feature-matching procedures, since the relative position components (and so specific object configurations with respect to position) were as disparate as this language (amplitude/ phase spectra) could guarantee. This control is to be contrasted with the conditions used in the previous two studies cited. In the Harvey and Gervais experiment, all textures were "in-phase," and so positively correlated (a control difficult to attain with naturally occurring textures). In the Coffin study, phase, defining the letter features (relative position), was confounded with amplitude factors. The issues associated with phase encoding and the phase-MTF/amplitudeMTF interactions need serious attention before we can derive, and represent in the context of digital image processing, the full two-dimensional filter for the perception of natural scene or texture attributes.

Although the information displayed in Experiment 1 differed from that of Experiment 2, both experiments address a common frequency/orientation filter mechanism. However, specific problems exist with using black-and-white displays which are not present in the gray-scale texture display apparatus. In particular, since the rectangular figures in Experiment 1 are defined by edges, one cannot readily modulate high-frequency information (by means other than optical blurring) and still maintain the binary valued nature of the stimulus. This is why only relative frequency components, such as the ratio of high/low frequency amplitudes, can be used (in this case they can fortunately be derived from Equation 15).

Consistent with the results of orientation illusion studies, it is concluded from Experiment 1 that as the frequency components decrease, orientation tuning width increases relative to the positions of the orientation detectors (assuming, for the moment, that there are a fixed number of orientation detectors at each frequency band). This filter characteristic has been applied to predicting discrimination between naturally occurring textures with some successjudged by the criterion of redundant information reduction. This criterion states that a filter matches an aspect of visual information processing if identical (behavioral), or better, predictions can be made with less information captured by the filter.

These textured stimuli lie between the one-dimensional textures of Harvey and Gervais and the alphabetic characters of Coffin, and the simple analyses conducted on discrimination performance indicate, in support of the Harvey and Gervais findings (as well as the author's and others'), that, for basic, simple nonfeature specific discrimination, the essential information codes lie within the image-amplitude spectra, and it remains the task of the experimenter to develop the correct filters that can tap the coding principles.

Although such filters may well apply to basic texture discrimination, image quality judgments, or other collective impressions of images, it seems clear that specific detection and recognition paradigms would seem to be less dependent on amplitude and more dependent on phase information and edge 
boundaries as such. However, just where this switch occurs in the processing of information from amorphous scenes (as in these experiments) to clearly defined objects has not been resolved.

In earlier studies with artificially generated textures, the author (Caelli, 1980; Caelli \& Julesz, 1978) has given many demonstrations of the conjecture that, in the absence of strong feature differences, textures with identical amplitude spectra are nondiscriminable. Yet the problem has remained as to what features of the amplitude spectra for perceivably different textures constitute the sufficient code for discrimination. In this paper, it is suggested that, to answer this question, it is more appropriate to explore the clam-shell filter than other, simple, onedimensional filters. Further parametric studies of this profile are being pursued, particularly in its digital form where $n$-orientation $\times$ m-frequency-specific units are individually configured in the spectrum (Caelli \& Harrison, Note 2).

\section{REFERENCE NOTES}

1. Caelli, T. M. The tilt illusion and orientation/spatial frequency sensitivity. Manuscript submitted for publication, 1981.

2. Caelli, T. M., \& Harrison, K. N-frequency channels $\times M$ orientation detectors: Information redundancy in visual image processing. Manuscript submitted for publication, 1981.

3. Caelli, T. M., \& Bevan, P. Sensitivity to two-dimensional spatial phase. Manuscript submitted for publication, 1981

\section{REFERENCES}

Beck, J. Perceptual grouping produced by changes in orientation and shape. Science, 1966, 154, 538-540.

Brettel, H., Caelli, T. M., Hilz, R., \& Rentschler, I. Amplitude and phase transmission in the human visual system: The two-dimensional case. Human Neurobiology, 1981, in press.

Brown, D. R., \& OWEN, D. M. The metrics of visual form: Methodological dyspepsia. Psychological Bulletin, 1967, 68, 243-259.

CAELl, T. M. Visual perception: Theory and practice. Oxford: Pergamon Press, 1981.

Caelli, T. M. Facilitative and inhibitory factors in texture discrimination. Biological Cybernetics, 1980, 39, 21-36.

Caelli, T. M., \& Julesz, B. On perceptual analyzers underlying visual texture discrimination: I. Biological Cybernetics, $1978,28,167-175$.
Caelli, T. M., \& Julesz, B. Psychophysical evidence for global feature processing in visual texture discrimination. Journal of the Optical Society of America, 1979, 69, 675-677.

Caelli, T. M., Julesz, B., \& Gilbert, E. On perceptual analyzers underlying visual texture discrimination: II. Biological Cybernetics, 1978, 39, 201-214.

Caelli, T. M., Preston, G., \& Howell, E. Implications of spatial summation models for processes of contour perception: A geometric perspective. Vision Research, 1978, 18, 723-734.

Coffin, S. Spatial frequency analysis of block letters does not predict experimental confusions. Perception \& Psychophysics, $1978,23,69-74$.

Cooley, W., \& Lohnes, P. Multivariate data analysis. New York: Wiley, 1971 .

DE VALoIs, R. Spatial tuning of LGN and cortical cells in monkey visual system. In H. Spekreijse \& L. Van der Tweel (Eds.), Spatial contrast. Amsterdam: Koninklijke Nederlandse, Academie van Wetenschappen, 1978.

Harvey, L., \& Gervais, M. Visual texture perception and Fourier analysis. Perception \& Psychophysics, 1978, 24, 534-542.

Julesz, B. Visual texture discrimination. IRE Transactions on Information Theory IT-8, 1962, 84-92.

JuLESz, B. Textons, the elements of texture perception, and their interactions. Nature, 1981, 290, 91-97.

Julesz, B., \& CAElli, T. M. On the limits of Fourier decompositions in visual texture perception. Perception, 1979, 8, 69-73.

KRUSKAL, J. Multidimensional scaling by optimizing goodness of fit to a nonmetric hypothesis. Psychometrika, 1964, 29, 1-27.

LEEUWENBERG, E. Quantification of certain visual pattern properties: Salience, transparency, similarity. In E. Leeuwenberg \& H. Buffart (Eds.), Formal theories of visual perception. New York: Wiley, 1978.

Mostafavi, H., \& Sakrison, D. Structure and properties of a single channel in the human visual system. Vision Research, $1976,16,957-968$

Movshon, J. A., \& Carpenter, C. Orientation specificity and spatial selectivity in human vision. Perception, 1973, 2, 53-80.

Richands, W., \& Polit, A. Texture matching. Kybernetik, 1974, 16, 155-162.

UTTAL, W. An autocorrelation theory of form detection. Hillsdale, N.J: Erlbaum, 1975.

\section{NOTES}

1. $\mathrm{cpd}=$ cycles per degree, the unit of spatial frequency; see Figure 2.

2. The textures used in this study were in-phase sinusoidal gratings embedded in random noise-essentially one-dimensional perturbations.

(Manuscript received January 5, 1981; revision accepted for publication October $13,1981$. ) 